\title{
ON OBSERVATION OF ULF ELECTROMAGNETIC SIGNALS FROM REMOTE EARTHQUAKES AND DISTRIBUTION OF THEIR SOURCES ON THE EARTH'S SURFACE
}

\section{B.V. Dovbnya $\mathbb{1}^{\otimes}$}

Borok Geophysical Observatory, Schmidt Institute of Physics of the Earth of the Russian Academy of Sciences, 142, Borok 152742, Russia

ABSTRACT. During long-term observations, the Borok and College Geophysical Observatories have registered ultralow-frequency (ULF) electromagnetic signals from remote earthquakes. We have analysed the characteristics of such signals that occur several minutes before a seismic event. Our analysis shows that the dynamic spectra of the signals from earthquakes that occurred in different regions are similar, although the earthquakes differ in magnitude and focal depth. We investigate and discuss daily and seasonal probabilities for the occurrence of ULF electromagnetic pulses. Attention is given to the uneven distribution of their sources (i.e. earthquakes) on the earth's surface. Our study shows that the ULF electromagnetic signals are clustered in separate zones and cells. When mapped, these clusters mark seismic electromagnetically active regions. In the northern hemisphere, a maximum cluster is found at latitudes $30-45^{\circ}$. In the longitudinal direction, two maximum clusters are located in the western sector. They are considered as the major and additional peaks (latitudes $120-150^{\circ}$ and $0-30^{\circ}$, respectively). Examples are given to illustrate earthquake precursors in various regions. Based on the analysis results, we conclude that the occurrence of ULF electromagnetic pulses before earthquakes is universal. These pulses need to be investigated in a more detail to clarify if an upcoming earthquake is detectable from such signals a few minutes before its occurrence, and whether it is possible, in principle, to use this information for safety alerts before seismic shaking arrives.

KEYWORDS: earthquake; electromagnetic signal; precursor; remote monitoring; spatial distribution

FUNDING: The study was financially supported by the Presidium of the Russian Academy of Sciences (program 28) and the Russian Foundation for Basic Research (project 19-05-00574), and carried out under State Assignment 01442014-00116.

\section{SHORT COMMUNICATION}

Correspondence: Boris V. Dovbnya, dovbnya@inbox.ru
Received: November 13, 2020

Revised: March 10, 2021

Accepted: March 11, 2021

FOR CITATION: Dovbnya B.V., 2021. On observation of ULF electromagnetic signals from remote earthquakes and distribution of their sources on the Earth's surface. Geodynamics \& Tectonophysics 12 (3), 563-569. doi:10.5800/GT-2021-12-3-0539 


\title{
О НАБЛЮДЕНИИ ИМПУЛЬСНЫХ УНЧ ЭЛЕКТРОМАГНИТНЫХ СИГНАЛОВ ОТ УДАЛЕННЫХ ЗЕМЛЕТРЯСЕНИЙ И РАСПРЕДЕЛЕНИИ ИХ ИСТОЧНИКОВ НА ЗЕМНОЙ ПОВЕРХНОСТИ
}

\author{
Б.В. Довбня
}

Геофизическая обсерватория «Борок» Института физики Земли им. О.Ю. Шмидта РАН, 152742, п. Борок, 142, Россия

АНнОТАЦИЯ. По данным многолетних наблюдений на двух разнесенных по широте и долготе геофизических обсерваториях «Борок» и «Колледж» проведен анализ свойств электромагнитных ультранизкочастотных (УНЧ) сигналов, обнаруживаемых от удаленных землетрясений за минуты до сейсмического события. Показано, что динамические спектры сигналов от землетрясений, происходящих в различных регионах Земли, подобны и повторяются при разных магнитудах и глубинах очага. Исследуются суточные и сезонные вероятности появления сигналов. Рассмотрено распределение источников обнаруженных сигналов, т.е. землетрясений, при которых они наблюдались, на земной поверхности. Показано, что они группируются в отдельные зоны и ячейки, отражая на карте регионы с сейсмоэлектромагнитной активностью. В Северном полушарии в распределении отмечается максимум в широтном направлении в интервале $30-45^{\circ}$, в долготном выделяются два максимума в западном секторе - основной в интервале $120-150^{\circ}$ и дополнительный в интервале $0-30^{\circ}$. Даются примеры, иллюстрирующие появление предвестников в различных регионах Земли. По результатам анализа делается вывод об универсальности процессов генерации импульсных сигналов перед землетрясениями и о принципиальной возможности краткосрочного предупреждения (за несколько минут) о предстоящем землетрясении.

КЛЮЧЕВЫЕ СЛОВА: землетрясение; электромагнитный сигнал; предвестник; дистанционное наблюдение; пространственное распределение

ФИНАНСИРОВАНИЕ: Работа выполнена при финансовой поддержке Программы № 28 Президиума РАН, проекта Российского фонда фундаментальных исследований 19-05-00574 и поддержана Гостемой 0144-201400116.

\section{1. ВВЕДЕНИЕ}

Настоящая работа является продолжением проводимых в геофизической обсерватории «Борок» исследований импульсных УНЧ электромагнитных сигналов, обнаруживаемых от удаленных землетрясений за минуты до сейсмического события [Dovbnya, 2014; Dovbnya et al., 2006, 2008, 2019].

Поиск предвестников землетрясений остается одной из основных проблем геофизики. Имеющиеся к настоящему времени экспериментальные данные (см. [Dovbnya et al., 2019] и приведенные в данной работе ссылки) указывают на возможность возникновения электромагнитных эффектов в УНЧ диапазоне перед сейсмическими событиями. И все же ситуация с предвестниками к настоящему времени остается неопределенной. Разные проявления электромагнитных аномалий и отсутствие повторяемости результатов вызывают сомнения в достоверности связи обнаруживаемых явлений с землетрясениями [Thomas et al., 2009a, 2009b]. Некоторые же из сообщений вызывают сомнения и оспариваются [Kosterin et al., 2015]. После обнаружения в 1964 г. эффекта генерации импульсных сигналов перед землетрясением на значительных удалениях от эпицентра [Moore, 1964] в литературе, как полагают авторы обзорной статьи «Есть ли новые открытия в поисках УНЧ-магнитных предвестников землетрясений?», «...никогда не было представлено убедительных доказательств в поддержку взаимосвязи между аномалией и землетрясением» [Masci, Thomas, 2015, c. 10289]. Тем не менее следует отметить работу [Malyshkov Yu.P., Malyshkov S.Yu., 2009], в которой отмечаются аналогичные наблюдения в поведении естественного импульсного электромагнитного поля Земли перед сейсмическими событиями.

В геофизической обсерватории «Борок» была предпринята попытка по данным непрерывных записей УНЧ вариаций электромагнитного поля Земли исследовать связь электромагнитных и сейсмотектонических процессов. В результате удалось обнаружить специфические УНЧ электромагнитные импульсы в диапазоне частот 0-5 Гц, наблюдаемые в выделенной и близкой временно́й окрестности землетрясений, которые по виду динамического спектра отличались от известных типов геомагнитных пульсаций [Dovbnya et al., 2006].

В данной работе продолжено исследование УНЧ электромагнитных импульсов, наблюдаемых от удаленных землетрясений. Анализируется суточно-сезонный ход вероятности появления сигналов, и рассмотрено пространственное распределение их источников, т.е. землетрясений, при которых сигналы наблюдались, на земной поверхности. Даются примеры, иллюстрирующие появление предвестников в различных регионах земной поверхности. Результаты обсуждаются.

\section{2. ИСХОДНЫЙ МАТЕРИАЛ И МЕТОДЫ АНАЛИЗА}

Анализ ультранизкочастотных излучений выполнялся по данным магнитных измерений в Геофизической обсерватории «Борок» Института физики Земли 
РАН (58. $1^{\circ}$ с.ш., $38.2^{\circ}$ в.д.) за период с 1973 по 1995 г. и в высокоширотной Геофизической обсерватории «Колледж» (64.9 ${ }^{\circ}$ с.ш., $148.0^{\circ}$ з.д.), расположенной в штате Аляска, США, за период с 1973 по 1977 г. Исходным материалом для анализа являлись записи УНЧ вариаций электромагнитного поля Земли. Для измерений использовался индукционный магнитометр с регистрацией на аналоговый магнитофон. Аналоговые записи подвергались спектрально-временному анализу с применением компьютерных программ. Строились динамические спектры колебаний (спектрограммы), на которых в координатах частота - время отражалась информация о переменном электромагнитном поле в анализируемом интервале. Для дальнейшего анализа отбирались сигналы, не коррелирующие с другими геофизическими явлениями, и им ставилось в соответствие, со статистической значимостью $\mathrm{p}=0.86$, ближайшее по каталогу Международного сейсмологического центра (http://www.isc.ac.uk/) землетрясение с конкретными географическими координатами эпицентра. Методика анализа подробно изложена в статье [Dovbnya et al., 2006] и дополнительно в работе [Dovbnya et al., 2019]. Ниже мы вначале дадим примеры, иллюстрирующие появление предвестников в различных регионах Земли. Затем исследуем суточно-сезонный ход импульсных сигналов, наблюдаемых от удаленных землетрясений, и рассмотрим пространственное распределение их источников, т.е. землетрясений, при которых сигналы наблюдались, на земной поверхности.

\section{3. РЕЗУЛЬТАТЫ НАБЛЮДЕНИЙ}

На рис. 1, а, б, даны примеры динамических спектров сигналов от землетрясений с разной магнитудой, произошедших в различных регионах земной поверхности (здесь и далее стрелками на рисунках отмечены моменты землетрясений. В подписях к рисункам приводятся следующие параметры землетрясений: мировое время, географические координаты, глубина $h$ в км, магнитуда M).

Из рисунка видно, что во всех случаях динамические спектры сигналов были подобны, наблюдались в одном частотном диапазоне и не зависели от параметров землетрясения, его магнитуды и глубины очага.

Интересно отметить повторяемость предвестников в землетрясениях, происходящих вслед за главным ударом в том же регионе. Это свойство, представляющее (a)

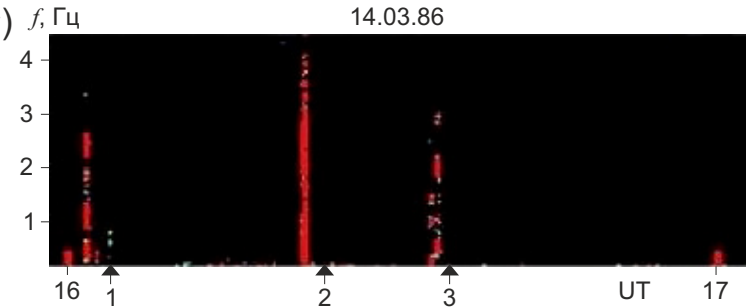

(б) $f, \Gamma 4$ 27.11 .76

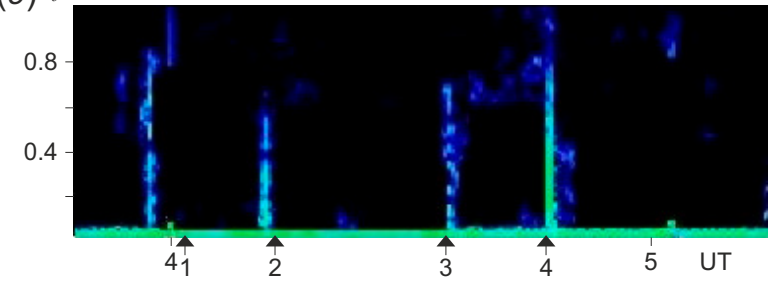

Рис. 1. Примеры динамических спектров сигналов в обсерваториях «Борок» (a) и «Колледж» (б). Моменты землетрясений отмечены стрелками.

Fig. 1. Dynamic spectra of ULF electromagnetic signals recorded by the Borok (a) and College (б) observatories. Earthquakes are marked by arrows.

\begin{tabular}{|c|c|c|c|c|c|}
\hline & $U T$ & $\varphi$ & $\lambda$ & $h$ & $M$ \\
\hline 1) & $16: 05: 13$ & -5.286 & 154.379 & 233 & 5.2 \\
\hline 2) & $16: 25: 45$ & 24.780 & 122.873 & 23 & 4.3 \\
\hline 3) & $16: 35: 27$ & -41.136 & 174.642 & 31 & 3.4 \\
\hline
\end{tabular}

(a)

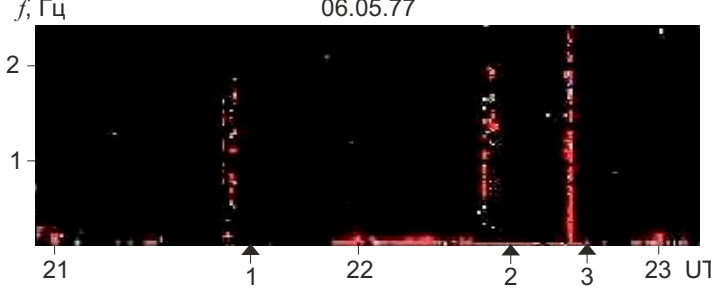

(б) $f, \Gamma\llcorner$

\begin{tabular}{ccrrcc} 
& $U T$ & $\varphi$ & \multicolumn{1}{c}{$\lambda$} & \multicolumn{1}{c}{$h$} & $M$ \\
1) & $4: 00: 09$ & -17.949 & -178.743 & 578 & 6.4 \\
2) & $4: 12: 38$ & 13.684 & 120.127 & 59 & 4.7 \\
3) & $4: 34: 17$ & 1.058 & -26.381 & 27 & 6.2 \\
4) & $4: 47: 22$ & 62.065 & -150.967 & 24 & -
\end{tabular}

Рис. 2. Примеры повторяемости предвестников по наблюдениям в обсерваториях «Колледж» (a) и «Борок» (б).

Fig. 2. Repeatability of precursors recorded by the College $(a)$ and Borok (б) observatories.

\begin{tabular}{|c|c|c|c|c|c|c|c|c|c|c|c|c|}
\hline & $U T$ & $\varphi$ & $\lambda$ & $h$ & $M$ & & & $U T$ & $\varphi$ & $\lambda$ & $h$ & $M$ \\
\hline 1) & $21: 37: 39$ & 46.796 & 153.83 & 35 & 5.9 & & 1) & $20: 03: 29$ & 38.415 & 26.723 & 11 & 4.1 \\
\hline a): 2) & $22: 28: 17$ & 46.700 & 154.20 & 40 & - & (б): & 2) & $20: 15: 20$ & 38.370 & 26.800 & 0 & 2.9 \\
\hline 3) & $22: 44: 17$ & 47.136 & 154.06 & 40 & 5.5 & & 3) & 20:17:51 & 38.314 & 26.797 & 10 & 3.3 \\
\hline
\end{tabular}


несомненный интерес, было подробно изучено в работе [Dovbnya, 2014]. Примеры появления предвестников в повторных толчках даны на рис. 2, а, б.

На рис. 3, а, б, даны примеры одновременного наблюдения сигналов в «Борок» и «Колледж».

Видно, что, несмотря на то, что обсерватории почти на 12 ч. разнесены по долготе и на $10^{\circ}$ по широте, предвестники на обеих станциях появляются практически одновременно и имеют подобную спектральную форму.
Перейдем к рассмотрению суточно-сезонного хода сигналов. На рис. 4, а, б, показано суточное распределение сигналов. Здесь мы ограничимся данными только обсерватории «Борок», поскольку объема имеющегося материала по «Колледжу» оказалось недостаточно для получения статистически надежных результатов.

На рис. 4, а, показано суточное распределение числа импульсов, усредненное по временам года. В вероятности их появления выделяются два максимума основной, который приходится на местные утренние

(a)

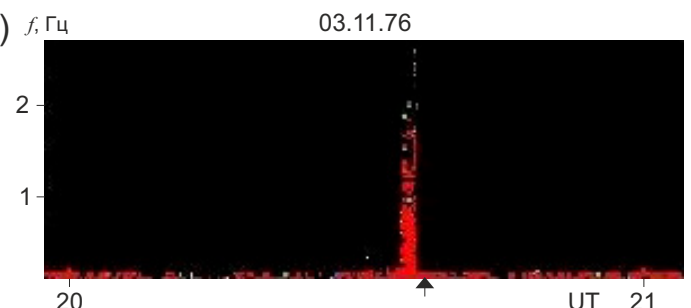

(б)

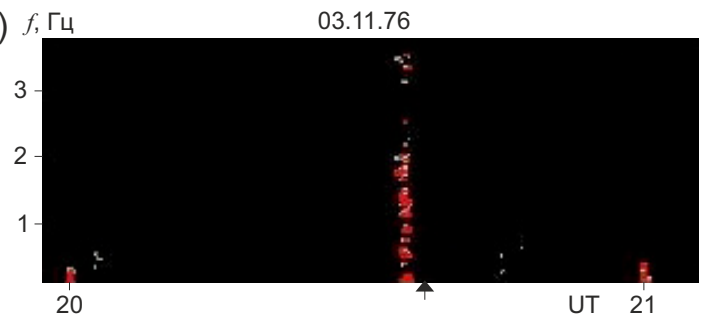

Рис. 3. Пример одновременного наблюдения сигнала в обсерваториях «Борок» (a) и «Колледж» (б).

Fig. 3. Simultaneous record of an ULF electromagnetic signal at the Borok $(a)$ and College (б) observatories.

$\begin{array}{ccccc}U T & \varphi & \lambda & h & M \\ 20: 37: 02 & 60.597 & -144.578 & 33 & 3.4\end{array}$

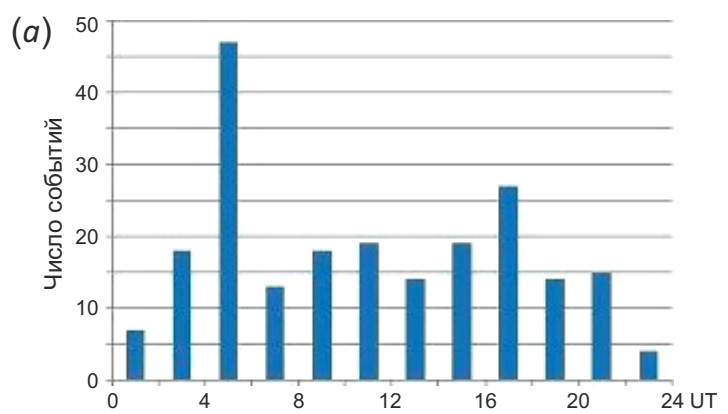

(б)

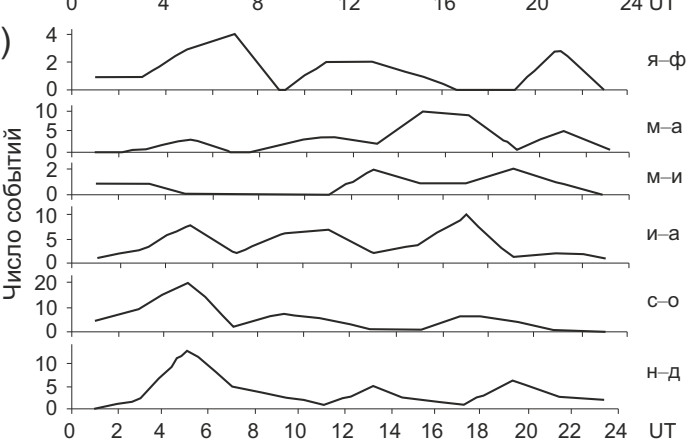

Рис. 4. Суточное распределение числа импульсов по данным обсерватории «Борок». (a) - усредненное по временам года, (б) в зависимости от сезона.

Fig. 4. Daily distribution of the number of ULF electromagnetic pulses recorded by the Borok Observatory. $(a)$ - seasonal average, (б) - in different seasons. 
часы (LT=UT+3), и дополнительный, который падает на местные вечерние часы. Форма вариаций может меняться в зависимости от сезона (рис. 4 , б) и отражает, по-видимому, влияние местных условий. В сезонном ходе частоты появления сигналов (рис. 5) отмечается максимум в весенний период, основное же возрастание числа событий приходится на зимние месяцы.

Наблюдение от удаленных землетрясений позволяло провести анализ распределения источников электромагнитного излучения (т.е. землетрясений, при которых наблюдались сигналы) на земной поверхности. На рис. 6, а, б, в, показаны результаты проведенного нами такого анализа. При построении использовались данные обсерваторий «Борок» (рис. 6, а, 228 событий) и «Колледж» (рис. 6, б, 78 событий). Красными кружками отмечены расположения обсерваторий на карте.

Анализ показывает широкое географическое и при этом неравномерное расположение источников излучения (рис. 6, а, б). Они группируются в отдельные зоны и ячейки, выделяя на фоне пространственного распределения по земному шару эпицентров землетрясений (рис. 6, в) регионы с проявлением сейсмоэлектромагнитной активности. Наблюдения на двух разнесенных по широте и долготе обсерваториях указывают, при разной статистике, на одни и те же зоны УНЧ электромагнитного излучения.

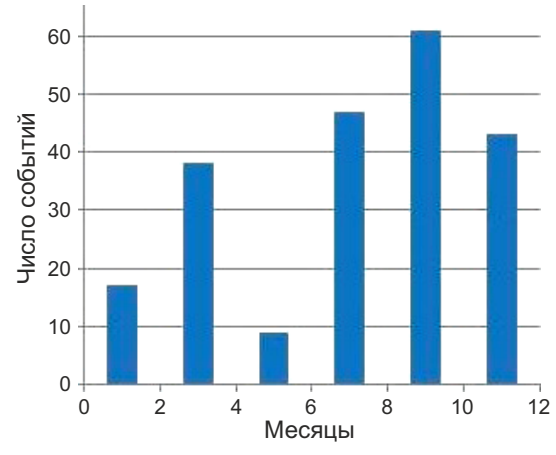

Рис. 5. Сезонный ход частоты появления сигналов.

Fig. 5. Seasonal variations in the occurrence frequency of ULF electromagnetic signals.

(a)

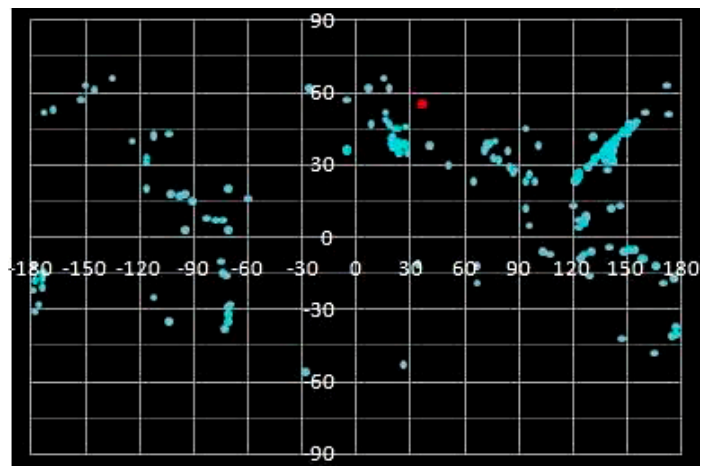

(б)

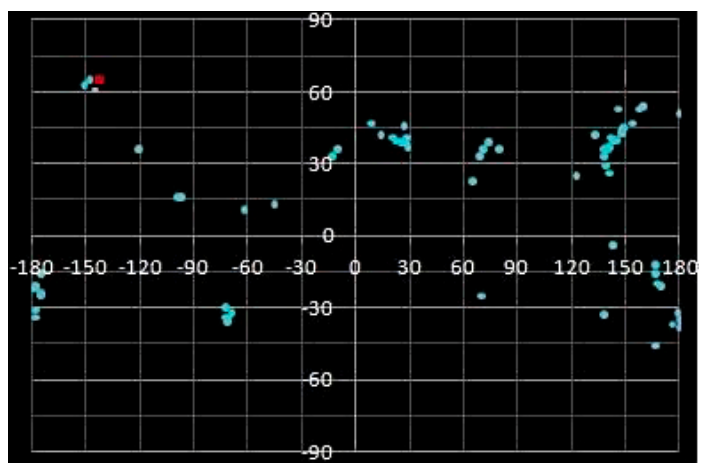

(в)

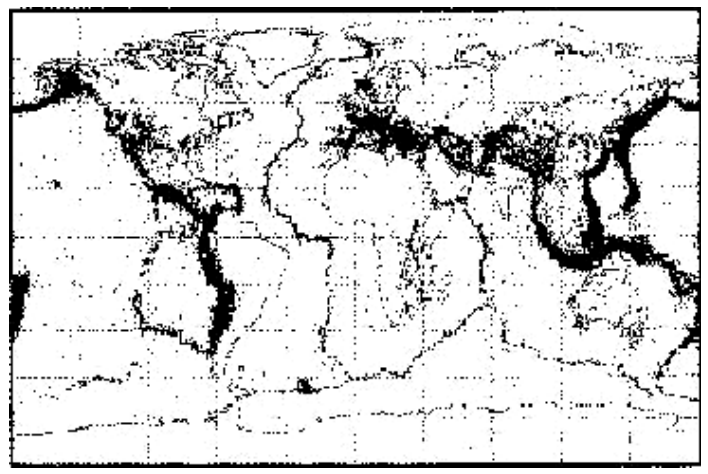

Рис. 6. Распределения источников УНЧ электромагнитных сигналов и эпицентров землетрясений на земной поверхности.

(a) - распределение источников по данным обсерватории «Борок», (б) - распределение источников по данным обсерватории «Колледж», ( 8 ) - распределение эпицентров землетрясений по земному шару.

Fig. 6. Surface distribution of the sources of ULF electromagnetic signals.

(a) - data of the Borok Observatoriy; (б) - data of the College Observatory; ( 8 ) - earthquake epicenters over the globe. 

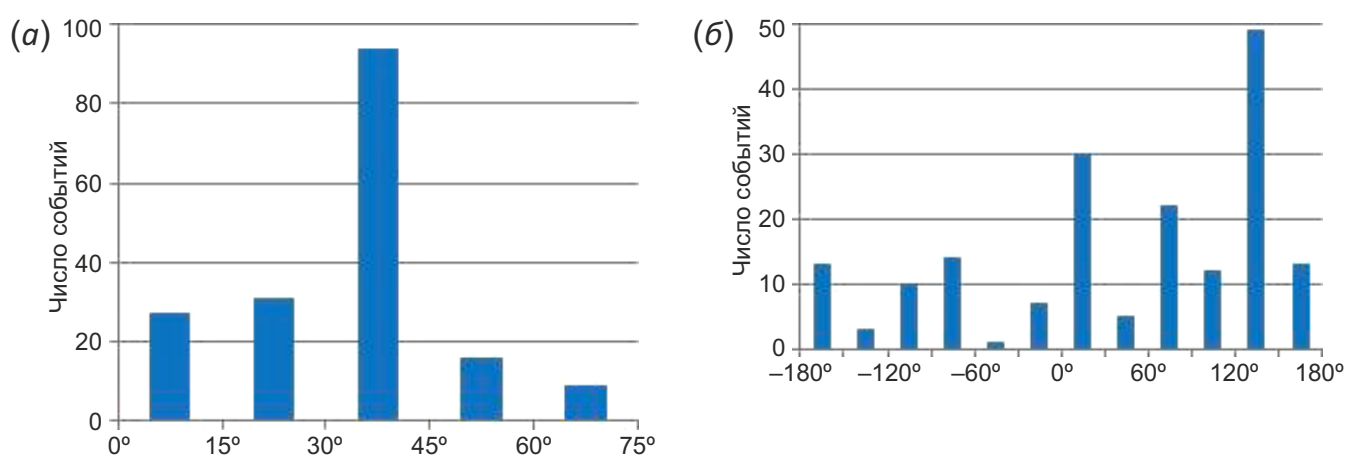

Рис. 7. Распределение источников УНЧ электромагнитных сигналов по широте (a) и долготе (б) для Северного полушария. Fig. 7. Distribution of the sources of ULF electromagnetic signals in the northern hemisphere by latitude ( $a$ ) and longitude (б).

В распределении источников сигналов по земному шару наблюдается явное различие по полушариям. Основная их часть приходится на Северное полушарие, где также заметна асимметрия в широтном и долготном направлении. На рис. 7 , а, б, показано распределение источников по широтным и долготным поясам Земли для Северного полушария. Широтные пояса брались шириной $15^{\circ}$ градусов, долготные - $30^{\circ}$. В распределении по широте выделяется четкий максимум в интервале $30-45^{\circ}$, в долготном направлении заметно проявляются два максимума в западном секторе: основной - в интервале $120-150^{\circ}$ и дополнительный - в интервале $0-30^{\circ}$.

\section{4. ОБСУЖДЕНИЕ И ЗАКЛЮЧЕНИЕ}

Итак, по данным многолетних наблюдений на двух разнесенных по широте и долготе обсерваториях показана реальность появления перед землетрясениями импульсных УНЧ электромагнитных сигналов и их регистрации на значительных расстояниях от эпицентра. Сигналы во всех случаях появлялись в одном частотном диапазоне (0-5 Гц) и в одном, выделенном относительно момента землетрясения, интервале времени (0-5 мин). По виду динамического спектра это были либо единичные, либо парные электромагнитные импульсы, частотно-временные характеристики которых были подобны и повторялись независимо от региона и параметров землетрясения. В работе [Dovbnya et al., 2019] в рамках модели землетрясений Рейда [Reid, 1910] обсуждается физика электромагнитных предвестников, где появление сигналов перед землетрясениями напрямую связывается с предшествующими землетрясению процессами.

Суточная и сезонная зависимость в появлении числа импульсов отражает влияние местных условий и условий на трассе распространения сигналов. Каналом для их распространения вдоль земной поверхности может служить ионосферный волновод, в котором сигналы способны распространяться от эпицентра на значительные расстояния. Дискретность динамического спектра импульсов, характерная для ионосферного распространения геомагнитных пульсаций [Dovbnya et al., 2014], не исключает такую возможность.
Анализ пространственного распределения источников электромагнитного излучения, доступный при дистанционной регистрации импульсных сигналов, показал их широкое географическое распространение по земному шару.

На основании полученных результатов можно сделать следующие выводы:

1. Электромагнитные сигналы перед землетрясениями - не случайный акт отдельного землетрясения, а проявление предваряющих землетрясение процессов, протекающих с преобразованием механической энергии в энергию электромагнитного излучения. Подобие форм динамических спектров и их повторяемость вне связи с параметрами землетрясений свидетельствуют об универсальности процессов генерации сигналов, предваряющих землетрясение.

2. Появление перед землетрясениями электромагнитных сигналов и широкое распространение их источников по земному шару создают возможность электромагнитного предупреждения (за несколько минут) о предстоящем землетрясении в большинстве сейсмоопасных регионов планеты.

\section{5. БЛАГОДАРНОСТИ}

Автор благодарит А.С. Потапова и Б.И. Клайна за полезное обсуждение проблем, затронутых в работе.

\section{6. ЛИТЕРАТУРА / REFERENCES}

Dovbnya B.V., 2014. Electromagnetic Precursors of Earthquakes and Their Recurrence. Geophysical Journal 36 (3), 160-165 (in Russian) [Довбня Б.В. Электромагнитные предвестники землетрясений и их повторяемость //Геофизический журнал. 2014. Т. 36. № 3. С. 160-165]. https:// doi.org/10.24028/gzh.0203-3100.v36i3.2014.116069.

Dovbnya B.V., Pashinin A.Yu., Rakhmatulin R.A., 2019. Short-Term Electromagnetic Precursors of Earthquakes. Geodynamics \& Tectonophysics. 10 (3), 731-740 (in Russian) [Довбня Б.В., Пашинин А.Ю., Рахматулин Р.А. Краткосрочные электромагнитные предвестники землетрясений // Геодинамика и тектонофизика. 2019. Т. 10. № 3. С. 731740]. https://doi.org/10.5800/GT-2019-10-3-0438.

Dovbnya B.V., Potapov A.S., Guglielmi A.V., Rakhmatulin R.A., 2014. On Influence of MHD Resonators upon Geomagnetic 
Pulsations. Geophysical Journal 36 (6), 143-152 (in Russian) [Довбня Б.В., Потапов А.С., Гульельми А.В., Рахматулин Р.А. О воздействии МГД резонаторов на геомагнитные пульсации // Геофизический журнал. 2014. Т. 36. № 6. C. 143-152]. https://doi.org/10.24028/gzh.0203-31 00.v36i6.2014.111053.

Dovbnya B.V., Zotov O.D., Mostryukov A.O., Shchepetnov R.V., 2006. Electromagnetic Signals Close in Time to Earthquakes. Izvestiya, Physics of the Solid Earth 42, 684689. https://doi.org/10.1134/S1069351306080052.

Dovbnya B.V., Zotov O.D., Shchepetnov R.V., 2008. Relationship of ULF Electromagnetic Waves with Earthquakes and Anthropogenic Impacts. Geophysical Studies 9, 3-23 (in Russian) [Довбня Б.В., Зотов О.Д., Щепетнов Р.В. Связь УНЧ электромагнитных волн с землетрясениями и антропогенными воздействиями // Геофизические исследования. 2008. Вып. 9. С. 3-23].

Kosterin N.A., Pilipenko V.A., Dmitriev E.M., 2015. On Global Ultralow Frequency Electromagnetic Signals Prior to Earthquakes. Geophysical Research 16 (1), 24-34 (in Russian) [Костерин Н.А., Пилипенко В.А., Дмитриев Э.М. 0 глобальных ультранизкочастотных электромагнитных сигналах перед землетрясениями // Геофизические исследования. 2015. Т. 16. № 1. С. 24-34].

Malyshkov Yu.P., Malyshkov S.Yu., 2009. Periodic Variations of Geophysical Fields and Seismicity, and Their Possible
Relationship with the Earth's Core Motion. Russian Geology and Geophysics 2, 152-172 (in Russian) [Малышков Ю.П., Малышков С.Ю. Периодические вариации геофизических полей и сейсмичности, их возможная связь с движением ядра Земли // Геология и геофизика. 2009. № 2. C. 152-172].

Masci F., Thomas J.N., 2015. Are There New Findings in the Search for ULF Magnetic Precursors to Earthquakes? Journal of Geophysical Research: Space Physics 120 (12), 10289-10304. https://doi.org/10.1002/2015JA021336.

Moore G., 1964. Magnetic Disturbances Preceding the 1964 Alaska Earthquake. Nature 203, 508-509. https:// doi.org/10.1038/203508b0.

Reid H.F., 1910. The Mechanics of the Earthquake. In: The California Earthquake of April 18, 1906. Report of the State Earthquake Investigation Commission. Vol. II. Carnegie Institution, Washington, $192 \mathrm{p}$.

Thomas J.N., Love J.J., Johnston M.J., 2009a. On the Reported Magnetic Precursor of the 1989 Loma Prieta Earthquake. Physics of the Earth and Planetary Interiors 173 (3-4), 207-215. https://doi.org/10.1016/j.pepi.2008. 11.014 .

Thomas J.N., Love J.J., Johnston M.J., Yumoto K., 2009b. On the Reported Magnetic Precursor of the 1993 Guam Earthquake. Journal of Geophysical Research: Solid Earth 36 (16). https://doi.org/10.1029/2009GL039020. 